\title{
ANISOTROPY OF MAGNETIC INTERACTIONS IN $\mathrm{HgFeSe}$
}

\author{
Z. Wilamowski, H. Przybylińska
}

Institute of Physics, Polish Academy of Sciences

Al. Lotników 32/46, 02-668 Warszawa, Poland

W. Joss AND M. GuILlot

MPI FKF/HML and CNRS/SHCI, BP 166X, 38042 Grenoble, France

Magnetic properties (susceptibility, high-field torque and magnetization) of cubic $\mathrm{HgFeSe}$ are analyzed. Van Vleck magnetism of $\mathrm{Fe}^{2+}$ is well evidenced. The energy splitting of the Fe levels in HgSe is shown to differ considerably from that in other II-VI compounds.

PACS numbers: 72.80 .Ey, $75.20 . \mathrm{Hr}$

The magnetic properties of $\mathrm{HgFeSe}$ were not, until now, systematically studied, and the preliminary results of susceptibility measurements reported by Lewicki et al. [1] called in question the Van Vleck magnetism of $\mathrm{Fe}^{2+}$ ions in $\mathrm{HgFeSe}$. As it turns out the low field susceptibility originates from both $2+$ and $3+$ charge states of $\mathrm{Fe}$ and the two components are difficult to unravel.

As it was shown by Testelin et al. [2] the magnetization of iron doped semimagnetic semiconductors is anisotropic. In this paper we report, apart from the usual studies of the magnetic field dependence of magnetization, the results of torque measurements for different values and orientations of the magnetic field. The latter measurements allow us to analyze the magnetic anisotropy much more accurately than the direct magnetization measurements.

The magnetization was measured at magnetic fields $H$ up to $20 \mathrm{~T}$ and temperatures in the range of 1.4 to $25 \mathrm{~K} \mathrm{for} \mathrm{Hg}_{1-x} \mathrm{Fe}_{x} \mathrm{Se}$ with iron content $x$ in the range of $0.003-0.05$. The magnetic field dependence of the magnetization measured at $4.2 \mathrm{~K}$ for $\boldsymbol{x}=0.01$ is shown in Fig. 1a. The inset shows the dependence of magnetization measured at $18 \mathrm{~T}$ on composition. In Fig. $1 \mathrm{~b}$ the temperature dependence of the susceptibility, $\chi_{g}$, for two samples with $x=0.01$ and 0.05 is shown. The susceptibility was determined from the slope of magnetization versus magnetic field in the range between 0 and $3 \mathrm{~T}$ to avoid the large experimental error encountered at very low fields. A typical Van Vleck (temperature independent) behaviour of the magnetic susceptibility can be observed in a very narrow range of temperatures $(T<5 \mathrm{~K})$ for low alloy compositions. At higher temperatures $\chi_{g}$ is governed by exchange interactions leading to the Curie-Weiss dependence 

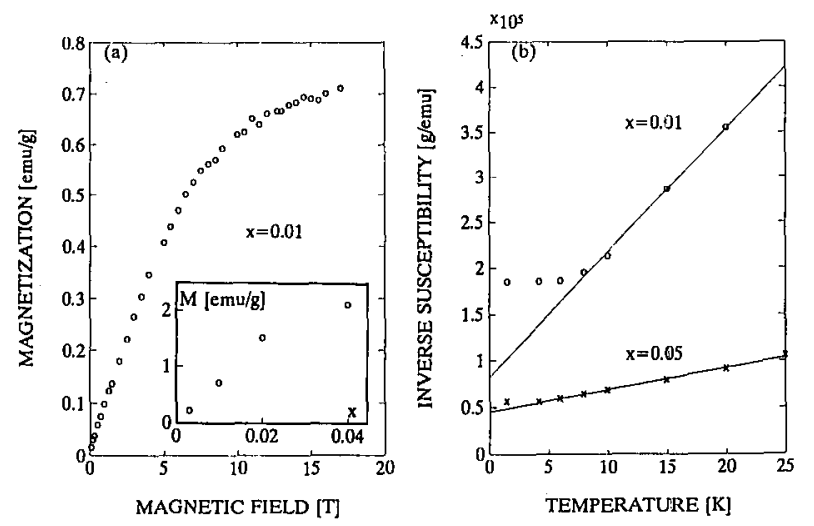

Fig. 1. (a) The magnetic field dependence of the magnetization measured at $4.2 \mathrm{~K}$ for $x=0.01$. The inset shows the dependence of magnetization measured at $18 \mathrm{~T}$ on composition. (b) The temperature dependence of the inverse susceptibility, $1 / \chi_{g}$, for two samples with $x=0.01(0)$ and $0.05(x)$. The parameters $\theta$ determined from the intersection of the solid lines with the temperature axis are $-6 \mathrm{~K}$ for $x=0.01$ and $-19.3 \mathrm{~K}$ for $x=0.05$.

of the susceptibility with $\Theta$ increasing with iron composition. On the other hand, the lower the iron content the greater the contribution of paramagnetic $\mathrm{Fe}^{3+}$ ions to the low-field susceptibility, in particular at low temperatures. For all those reasons the magnetic susceptibility of $\mathrm{HgFeSe}$ is very complex, so in this paper we concentrate on magnetization and torque measurements at high magnetic fields.

The symmetry conditions require that in a cubic crystal at low magnetic fields the magnetization is linear in $H$ and isotropic, thus the torque is equal to zero. At increased magnetic fields (and low temperatures) the magnetization ceases to be linear and an anisotropic magnetization component appears, proportional to the 3rd power of the magnetic field, leading to a simultaneous appearance of the torque with a 4th power magnetic field dependence. The general expression for the fourth order angular dependence of the torque is given by a derivative $\mathrm{d} \Phi / \mathrm{d} \alpha$ of the scalar function of cubic symmetry: $\Phi=\left(l^{2} m^{2}+m^{2} n^{2}+n^{2} l^{2}\right)$, where $(l m n)$ are the direction cosines with respect to the cubic axes and $\alpha$ denotes the angle around the rotation axis. For the magnetic field rotated in the (110) plane $\mathrm{d} \Phi / \mathrm{d} \alpha$ is expressed by $F(\alpha)=(3 \sin 4 \alpha-2 \sin 2 \alpha) / 4$, where $\alpha$ is the angle between $\boldsymbol{H}$ and the $[1 \overline{1} 0]$ axis. The experimental dependence of the torque measured along the [110] axis on $\alpha$ is shown in Fig. 2a for $H=19 \mathrm{~T}$ and two compositions $(x=0.003$ and 0.01$)$. The measurements were performed at $4.2 \mathrm{~K}$. It can be seen that at high magnetic fields the fourth order term $F(\alpha)$ dominates. However, for quantitative description (solid lines in Fig. 2a) a lower order contribution to the torque, proportional to $H^{2} \sin 2\left(\alpha-\varphi_{\text {dep }}\right)$, has to be included. This contribution is an effect of depolarization connected with the non-spherical macroscopic symmetry of the sample. 

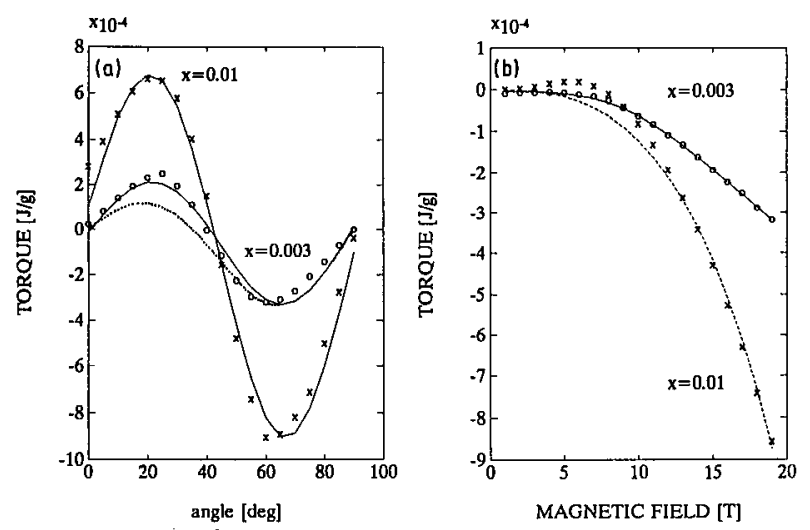

Fig. 2. (a) The angular dependence of the torque measured along the [110] axis for

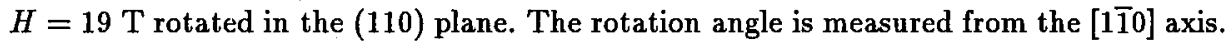
Experimental data are indicated with symbols, (o) for the sample with $x=0.003$ and $(x)$ for $x=0.01$. The measurements were performed at $4.2 \mathrm{~K}$. The dotted line shows the angular dependence calculated with the fourth order function $F(\alpha)$, solid lines show the dependence calculated with depolarization effects of the form $\sin 2\left(\alpha-\varphi_{\text {dep }}\right)$ included. (b) The magnetic field dependence as measured for the same samples at $\alpha=65^{\circ}$. Solid lines show the dependence calculated for $6 \lambda^{2} / \Delta=3.4 \mathrm{meV}$ without the second-order term resulting from depolarization effects, hence the discrepancy which can be observed for $x=0.01$ at low magnetic fields.

In order to describe the magnetic field dependence of the torque at high magnetic fields an analysis based only on symmetry considerations is not sufficient and the total Hamiltonian for the $\mathrm{Fe}^{2+}$ ion, $\mathcal{H}=\mathcal{H}_{\mathrm{cr}}+\lambda \boldsymbol{L} \cdot \boldsymbol{S}+\mu_{\mathrm{B}}(\boldsymbol{L}+2 \boldsymbol{S}) \cdot \boldsymbol{H}$, has to be considered. The ground state of a single $\mathrm{Fe}^{2+}$ ion in a crystal field of $T_{d}$ symmetry is an orbital doublet ${ }^{5} E$, split by spin-orbit interaction with the excited orbital triplet $\left({ }^{5} T_{2}\right)$ into five almost equally spaced (at $H=0$ ) levels, denoted according to their symmetries as $\Gamma_{1}, \Gamma_{4}, \Gamma_{3}, \Gamma_{5}$, and $\Gamma_{2}$, the lowest being the $\Gamma_{1}$ singlet state. Within the accuracy of second-order perturbation theory the splitting is equal to $6 \lambda^{2} / \Delta$, where $\Delta$ denotes the crystal field splitting. At low temperatures only the lowest state can be considered (since $k T \ll 6 \lambda^{2} / \Delta$ ). For low magnetic fields $\left(g \mu_{\mathrm{B}} H \ll 6 \lambda^{2} / \Delta\right)$ its energy can be calculated by perturbation theory and up to the fourth order takes the form

$$
E=-\frac{8\left(\mu_{\mathrm{B}} H\right)^{2} a^{2}}{\left(6 \lambda^{2} / \Delta\right)}+\frac{32\left(\mu_{\mathrm{B}} H\right)^{4} a^{2}}{\left(6 \lambda^{2} / \Delta\right)^{3}}\left[a^{2}+\left(3 a^{2}-f^{2}\right) \Phi\right]
$$

where $a \cong 1-4 \lambda / \Delta$ and $f \cong 1-2 \lambda / \Delta$. The torque value along the rotation axis can be expressed by $T=-\partial E / \partial \alpha$ (where $\alpha$ denotes the rotation angle), while the Van Vleck magnetic moment is given by $\boldsymbol{m}=-\partial E / \partial \boldsymbol{H}$.

By comparing the experimental results with theory we obtain $3.4 \mathrm{meV}$ for the level splitting $6 \lambda^{2} / \Delta$. This value describes well both the susceptibility and the magnetic field dependence of the torque (Fig. 2b). One should bear in mind, however, that though for the analysis of magnetic susceptibility even the first term 
in Eq. (1) suffices, to analyze the magnetic field dependence of the torque numerical calculations had to be performed. We would like to stress that the $3.4 \mathrm{meV}$ value is significantly larger than that reported for $\mathrm{Fe}^{2+}$ in other selenides. In consequence, the lower than usual Van Vleck susceptibility can be easily obscured by other effects.

\section{References}

[1] A. Lewicki, J. Spałek, A. Mycielski, J. Phys. C 20, 2005 (1987).

[2] C. Testelin, A. Mauger, C. Rigaux, M. Guillot, A. Mycielski, Solid State Commun. 71, 923 (1989); see also C. Testelin, Ph.D. Thesis, Université Pierre et Marie Curie, Paris, 1991. 